\section{Gene Transfer of Glutamic Acid Decarboxylase Reduces Neuropathic Pain}

Shuanglin Hao, MD, PhD, ${ }^{1}$ Marina Mata, MD, ${ }^{1}$ Darren Wolfe, PhD, ${ }^{2}$ Joseph C. Glorioso, PhD, ${ }^{2}$ and David J. Fink, MD ${ }^{1}$

We tested whether transfer of the gene coding for glutamic acid decarboxylase to dorsal root ganglion using a herpes simplex virus vector to achieve release of GABA in dorsal horn would attenuate nociception in this condition. Subcutaneous inoculation of a replication-defective herpes simplex virus vector expressing glutamic acid decarboxylase (vector QHGAD67) 7 days after selective L5 spinal nerve ligation reversed mechanical allodynia and thermal hyperalgesia; the antiallodynic effect lasted 6 weeks and was reestablished by reinoculation. QHGAD67 inoculation also suppressed induction of c-Fos and phosphorylated extracellular signal-regulated kinase 1 and 2 in the spinal cord.

Ann Neurol 2005;57:914-918

Peripheral neuropathic pain is a common and difficult to treat concomitant of polyneuropathy or structural nerve injury. Opioids are relatively ineffective, and their use is limited by side effects. Antidepressants and anticonvulsants have demonstrated efficacy in randomized controlled trials but provide only $50 \%$ relief in less than half of patients treated. ${ }^{1}$ Among the complex mechanisms underlying neuropathic pain, partial nerve injury results in a selective loss of GABAergic inhibitory synaptic currents in spinal $\operatorname{cord}^{2}$ that contribute to abnormal pain sensitivity and the phenotypic features of the neuropathic pain syndrome. GABAergic agents have not been widely used in the treatment of neuropathic pain because the therapeutic window of these agents is modest and the dose is limited by side effects.

Gene transfer represents a novel and useful means to target expression of peptides to focal sites within the

From the ${ }^{1}$ Department of Neurology, University of Michigan Health System and Ann Arbor VA Healthcare System, Ann Arbor, MI; and ${ }^{2}$ Department of Molecular Genetics and Biochemistry, University of Pittsburgh, Pittsburgh, PA.

Received Nov 30, 2004, and in revised form Mar 4 and Mar 11, 2005. Accepted for publication Mar 11, 2005.

Published online May 23, 2005, in Wiley InterScience (www.interscience.wiley.com). DOI: 10.1002/ana.20483

Address correspondence to Dr Fink, Department of Neurology, University of Michigan Health System, 1500 E. Medical Center Drive, Room 1914 TC, Ann Arbor, MI 48109-0316.

E-mail: djfink@umich.edu nervous system. We have shown that transduction of sensory neurons of the dorsal root ganglion (DRG) by footpad inoculation with herpes simplex virus (HSV)based vectors can be used to achieve a regional antinociceptive effect. HSV vectors coding for proenkephalin or the glial cell-derived neurotrophic factor produce an antihyperalgesic and antiallodynic effect in rodent models of inflammatory pain, neuropathic pain, and pain resulting from cancer in bone, ${ }^{3-6}$ and an HSVvector expressing glutamic acid decarboxylase (GAD) provides an analgesic effect in the central neuropathic pain syndrome resulting from spinal cord injury. ${ }^{7}$ In this study, we examined the antinociceptive effect of GAD expressed from an HSV-based vector in the spinal nerve ligation (SNL) model of neuropathic pain in the rat.

\section{Materials and Methods}

The nonreplicating HSV vector QHGAD67, which is defective in expression of the HSV immediate early genes $I C P 4$, ICP22, ICP27, and ICP47 and contains the human GAD67 gene under the control of the human cytomegalovirus immediate early promoter in the $\mathrm{U}_{\mathrm{L}} 41$ locus, has been described previously. ${ }^{7}$ The control vector QOZHG is defective in the same HSV genes but contains the green fluorescent protein and Escherichia coli lacZ reporter genes. ${ }^{7}$

Male Sprague-Dawley rats weighing 225 to $250 \mathrm{gm}$ underwent selective L5 SNL, as described previously, ${ }^{5}$ with the approval of the University Committee on Use and Care of Animals. One week after SNL, $30 \mu$ l of vector (either QHGAD67 or QOZHG, $4 \times 10^{8}$ plaque-forming units per milliliter) was injected subcutaneously in the plantar surface of the left hind paw, ipsilateral to the ligation. Mechanical allodynia induced by SNL was determined by assessing the response of paw withdrawal to von Frey hairs of graded tensile strength as described previously, ${ }^{5,8}$ with a tactile stimulus producing a $50 \%$ likelihood of withdrawal determined using the up-down method. ${ }^{9}$ Thermal hyperalgesia was determined using a Hargreaves apparatus, ${ }^{10}$ recording the time to withdrawal from a radiant thermal stimulus positioned directly under the hind paw.

\section{Results}

After L5 SNL, rats displayed a significant decrease in the magnitude of the mechanical stimulus necessary to evoke a brisk withdrawal response to von Frey hair stimulation (Fig 1A) and a significant reduction in latency to withdraw from a heat stimulus (thermal hyperalgesia; see Fig 1B). Rats inoculated with QHGAD67 showed a statistically significant increase in mechanical threshold beginning 1 week after inoculation. The antiallodynic effect of QHGAD67-mediated GABA expression was sustained and continuous, lasting 5 to 6 weeks and peaking at 2 weeks after inoculation (see Fig 1A). The peak value of mechanical threshold, $8.6 \mathrm{gm}$, was close to the preoperative value. By 7 weeks after inoculation, the antiallodynic effect of 


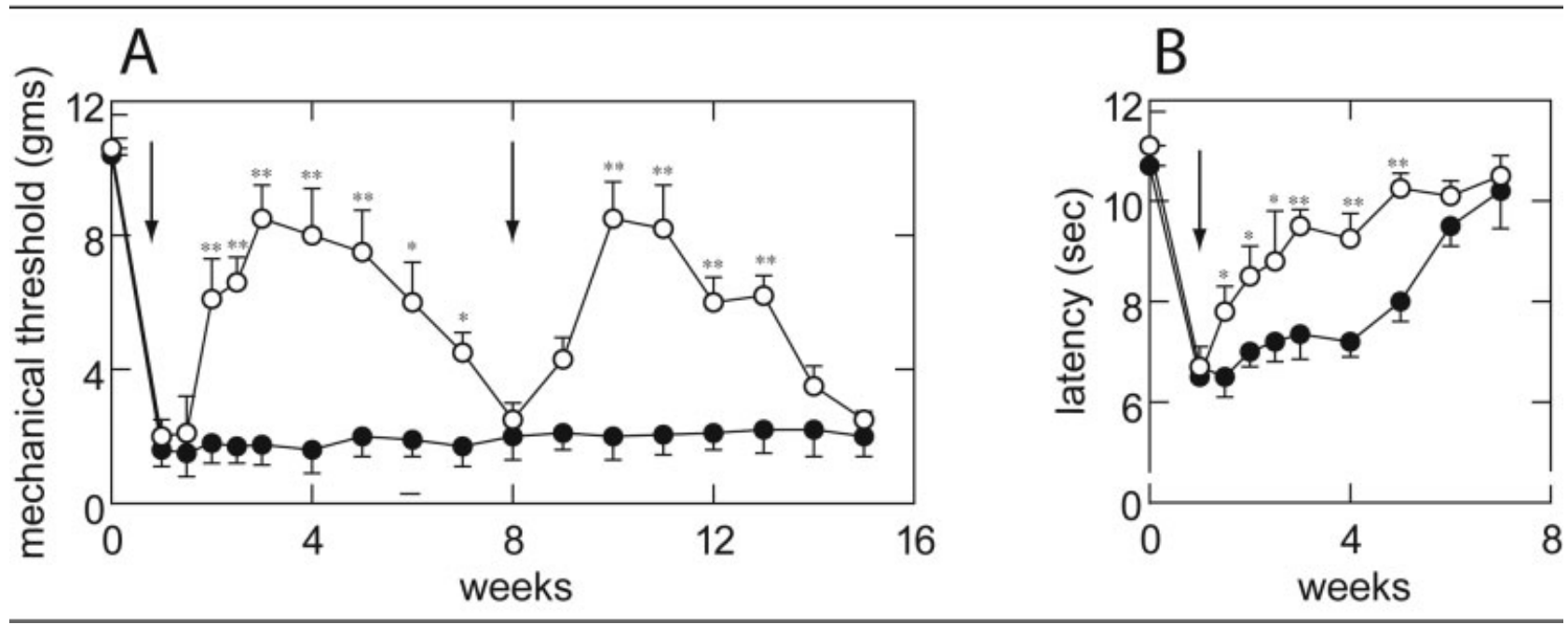

Fig 1. Antinociceptive effect of QOGAD67 in neuropathic pain. (A) L5 spinal nerve ligation (SNL) caused a significant decrease in the threshold to tactile stimulation, which persisted for more than 4 months. Subcutaneous inoculation of QHGAD67 (arrow) produced an antiallodynic effect reflected in an increase in the mechanical threshold. Reinoculation of QHGAD67 7 weeks after the initial inoculation (arrow) reestablished the antiallodynic effect. Results are expressed as mean \pm standard error of the mean. (open circles) QHGAD67; (closed circles) QOZHG; ${ }^{*} \mathrm{p}<0.05 ;{ }^{* *} \mathrm{p}<0.01 ; n=8$ animals per group. (B) L5 SNL also caused a significant thermal hyperalgesia, which persisted for 6 weeks. Inoculation with QHGAD67 (arrow), but not QOZHG, reversed the thermal hyperalgesia induced by spinal nerve injury. ${ }^{*} \mathrm{p}<0.05 ;{ }^{* *} \mathrm{p}<0.01$ versus QOZHG-inoculated; $n=8$ animals per group. The statistical significances of the differences were determined by analysis of variance (StatView 5.2; SAS Institute, Cary, NC) corrected for the number of post hoc comparisons using Scheffé's F test.

vector transduction disappeared, and the mechanical threshold of QHGAD67-injected rats was identical to that of control rats. Reinoculation into the same paw with the same dose of QHGAD67 reestablished the antiallodynic effect (see Fig 1A).

SNL induced a decrease in the thermal latency from 10.7 to 6.5 seconds, which lasted 3 weeks before gradually recovery. Rats inoculated with QHGAD67 showed a statistically significant increase in thermal latency in the ipsilateral paw beginning 1 week after inoculation (see Fig 1B), an effect that was sustained and continuous, lasting 3 to 4 weeks (see Fig 1B). Shamoperated animals had no change in mechanical threshold or thermal latency (data not shown).

Expression of c-Fos and phosphorylated extracellular signal-regulated kinase 1 and 2 (p-ERK1/2) induced by gentle touch is one indirect biological marker of nociceptive processes. ${ }^{11}$ Three weeks after SNL, gentle touch was applied once every 4 seconds for 10 minutes, with the flat surface of the experimenter's thumb to the rat's paw, and the number of immunoreactive cells (anti-c-Fos or anti-p-ERK1/2 antibodies; Santa Cruz Biotechnology, Santa Cruz, CA) detected avidinbiotin horseradish peroxidase followed by nickelenhanced diaminobenzidine (Vector Laboratories, Burlingame, CA). ${ }^{12}$ The number of Fos-LI-positive neurons was substantially increased ipsilateral to SNL compared with sham-operated control rats (Fig 2), and inoculation of vector QHGAD67 significantly reduced the number Fos-LI-positive neurons in laminae I-VI (see Fig 2). p-ERK1/2 expression in laminae I and II was also increased in rats after gentle touch stimulation with SNL, and that increase was blocked in animals inoculated with QHGAD67 (Fig 3).

\section{Discussion}

These results demonstrate that subcutaneous inoculation of an HSV vector expressing GAD to transduce DRG in vivo attenuated the behavioral manifestations of mechanical allodynia and thermal hyperalgesia in a model of peripheral neuropathic pain; the effect on behavior was confirmed by histological measures showing a block in the induction of expression of c-Fos and p-ERK1/2 in the ipsilateral spinal dorsal horn. We have demonstrated previously that transduction of primary sensory neurons with QHGAD67 results in the release of GABA from those cells in vitro and from sensory nerve terminals in spinal cord in vivo. ${ }^{7}$ Subcutaneous inoculation of the vector results in transduction of both large and small neurons in the DRG, as determined by immunocytochemical detection of GAD expression. ${ }^{7}$ GABA release from transduced cells is constitutive and is mediated by the GABA transporter type 1 (GAT-1). In the spinal cord hemisection model of central neuropathic pain, transduction of DRG by subcutaneous inoculation of QHGAD67 in both hind paws reduces below-level mechanical allodynia and thermal hyperalgesia. ${ }^{7}$ This study is the first application of this vector to peripheral neuropathic pain.

In the spinal cord, GABA is found primarily in in- 

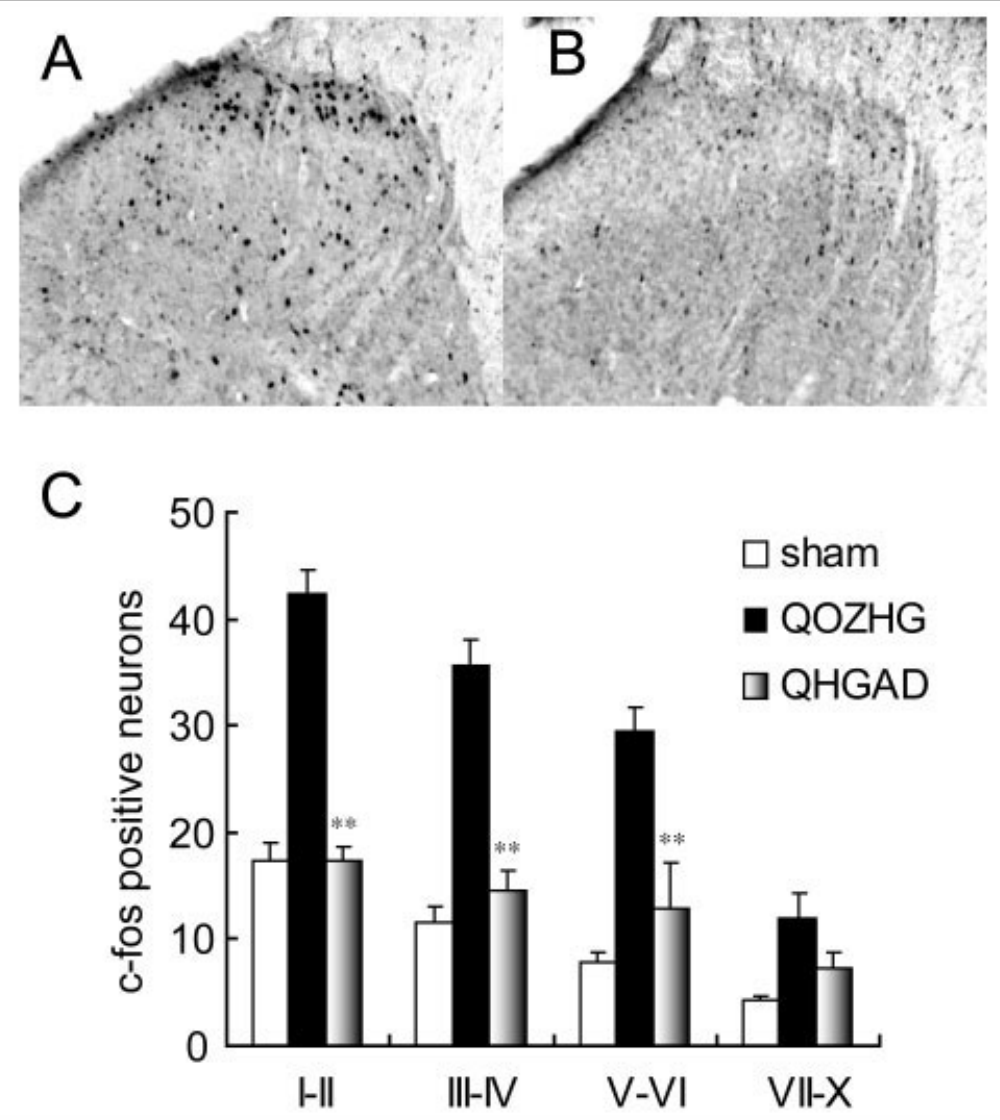

Fig 2. Effect of QHGAD67 on Fos-LI in dorsal horn. (A) Fos-LI in dorsal horn induced by 10 minutes of gentle tactile stimulation was markedly increased in rats inoculated with QOZHG 1 week after spinal nerve ligation (SNL) and tested 2 weeks later (3 weeks after SNL). (B) This increase was blocked in rats with SNL that had been inoculated with QHGAD67 1 week after SNL and tested 2 weeks later ( 3 weeks after SNL), and it was found in laminae I-VI of dorsal horn. Results are expressed as mean \pm standard error of the mean. ${ }^{* *} \mathrm{p}<0.01 ; n=5$ animals per group. The difference between sham-operated and SNL animals inoculated with QOZHG was also statistically significant $(\mathrm{p}<0.01)$.

terneurons of the superficial dorsal horn predominantly in laminae II and III that form axoaxonic synapses on primary afferent terminals and axodendritic synapses on projection neurons. Tonic GABAergic inhibition plays an important role in normal sensory processing, as indicated by increased behavior responsiveness ${ }^{13}$ and electrophysiological activity in spinal nociceptive neurons ${ }^{14}$ after spinal administration of the $\mathrm{GABA}_{\mathrm{A}}$ antagonist bicuculline to normal animals and by the development of mechanical allodynia after spinal administration of the $\mathrm{GABA}_{\mathrm{B}}$ receptor antagonist CGP35348. ${ }^{15}$

The mechanical allodynia that results from selective SNL can be reversed by the $\mathrm{GABA}_{\mathrm{B}}$ agonist baclofen ${ }^{16}$ or the $\mathrm{GABA}_{\mathrm{A}}$ agonist muscimol, ${ }^{17}$ and both mechanical allodynia and thermal hyperalgesia after spinal nerve injury are reversed by the $\mathrm{GABA}_{\mathrm{A}}$ agonist 4,5,6,7-tetrahydroisoxazolo [5,4-c] pyridin-3-ol (isoguvacine) and baclofen. ${ }^{18}$ These findings are consistent with observations of loss of GABA activity in dorsal horn after chronic constriction injury of sciatic nerve, ${ }^{19}$ a decrease in $\mathrm{GABA}_{\mathrm{A}}$ receptor-mediated inhibitory postsynaptic potentials, reduction of GAD65, and evidence of apoptosis in dorsal horn in both the chronic constriction and spared nerve injury models of neuropathic pain. ${ }^{2}$ Transgene-mediated GABA released in the dorsal horn acts at both $\mathrm{GABA}_{\mathrm{A}}$ and $\mathrm{GABA}_{\mathrm{B}}$ receptors. As we have demonstrated previously, the antinociceptive effect produced by QHGAD67-mediated GABA release can be partially blocked by the $G_{A B A}$ antagonist bicuculline or by the $\mathrm{GABA}_{\mathrm{B}}$ antagonist phaclofen. $^{7}$

GABAergic therapy for neuropathic pain has had limited success. Gene transfer offers the possibility of targeted alteration in the neurotransmitter phenotype to produce the local release of selected neurotransmitters. Among the many viral and nonviral vectors that may be used for gene transfer, HSV, because of its natural neurotropism and high-affinity retrograde transport in sensory neurons, is uniquely suited for modifying neurotransmitter release in dorsal horn after subcutaneous inoculation. ${ }^{20}$ We have found previously 

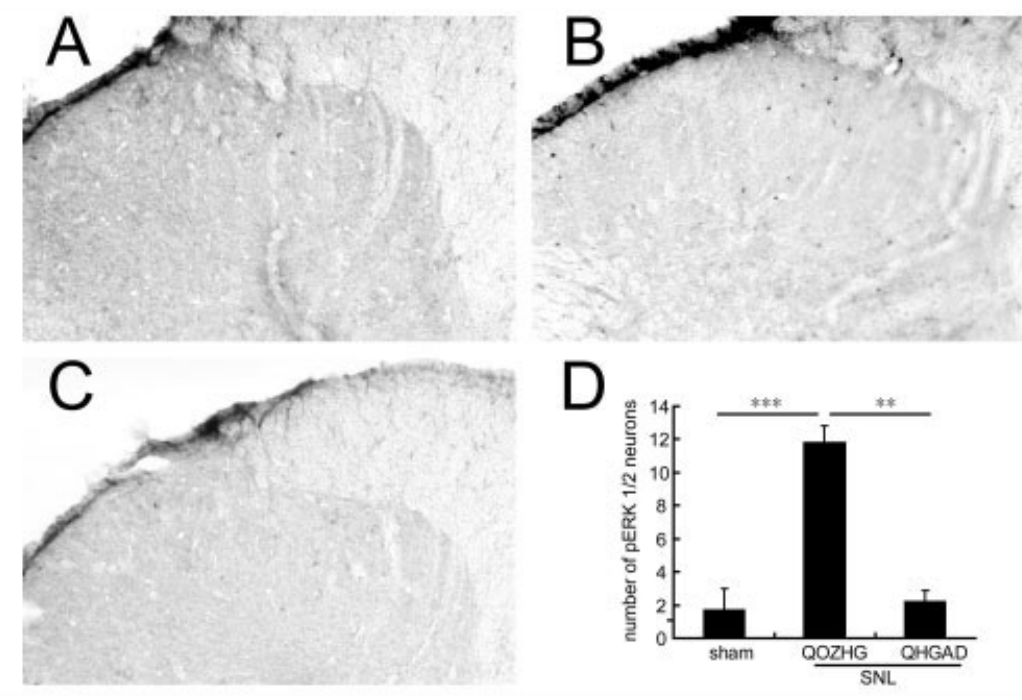

Fig 3. Effect of QHGAD67 on the phosphorylated extracellular signal-regulated kinase 1 and 2 (p-ERK1/2) expression in dorsal horn. $p$-ERK is not induced by 10 minutes of gentle tactile stimulation in sham-operated animals (A), but it is substantially induced after spinal nerve ligation (SNL) in animals inoculated with QOZHG (B). Touch-induced expression of p-ERK1/2 was suppressed in animals inoculated with QHGAD67 (C), confirmed by counts of p-ERK1/2-positive neurons in the dorsal horn (D). Results are expressed as mean \pm standard error of the mean. ${ }^{* *} \mathrm{p}<0.01 ;{ }^{* * *} \mathrm{p}<0.001 ; n=5$ animals per group.

that HSV-mediated transfer of proenkephalin produces a sustained antiallodynic effect in the SNL model of neuropathic pain, ${ }^{5}$ and that similar results can be achieved with a vector expressing glial cell-derived neurotrophic factor. ${ }^{6}$ The magnitude of the antiallodynic effect achieved with the GAD67-expressing vector is substantially greater than that produced by either the proenkephalin- or glial cell-derived neurotrophic factor-expressing vectors. We propose that this differential effect reflects the role of reduced spinal GABAergic tone in the pathogenesis of neuropathic pain and the selective ability of QHGAD67 to correct that deficit locally. The results suggest that this novel approach may prove useful in the treatment of neuropathic pain.

This study was supported by grants from the NIH (National Institute of Neurological Disorders and Stroke, NS44507, NS38850, D.J.F., NS43247, M.M.) and the Department of Veterans Affairs (M.M., D.J.F.).

We thank V. Thakur and S. Liu for their excellent technical assistance.

\section{References}

1. Sindrup SH, Jensen TS. Efficacy of pharmacological treatments of neuropathic pain: an update and effect related to mechanism of drug action. Pain 1999;83:389-400.

2. Moore KA, Kohno T, Karchewski LA, et al. Partial peripheral nerve injury promotes a selective loss of GABAergic inhibition in the superficial dorsal horn of the spinal cord. J Neurosci 2002;22:6724-6731.

3. Wilson SP, Yeomans DC, Bender MA, et al. Antihyperalgesic effects of infection with a preproenkephalin-encoding herpes virus. Proc Natl Acad Sci U S A 1999;96:3211-3216.
4. Braz J, Beaufour C, Coutaux A, et al. Therapeutic efficacy in experimental polyarthritis of viral-driven enkephalin overproduction in sensory neurons. J Neurosci 2001;21:7881-7888.

5. Hao S, Mata M, Goins W, et al. Transgene-mediated enkephalin release enhances the effect of morphine and evades tolerance to produce a sustained antiallodynic effect. Pain 2003;102: $135-142$.

6. Hao S, Mata M, Wolfe D, et al. HSV-mediated gene transfer of the glial cell derived neurotrophic factor (GDNF) provides an anti-allodynic effect in neuropathic pain. Mol Ther 2003;8: 367-375.

7. Liu J, Wolfe D, Hao S, et al. Peripherally delivered glutamic acid decarboxylase gene therapy for spinal cord injury pain. Mol Ther 2004;10:57-66.

8. Chaplan SR, Bach FW, Pogrel JW, et al. Quantitative assessment of tactile allodynia in the rat paw. J Neurosci Methods 1994;53:55-63.

9. Dixon WJ. Efficient analysis of experimental observations. Annu Rev Pharmacol Toxicol 1980;20:441-462.

10. Hargreaves K, Dubner R, Brown F, et al. A new and sensitive method for measuring thermal nociception in cutaneous hyperalgesia. Pain 1988;32:77-88.

11. Catheline G, Le Guen S, Besson JM. Intravenous morphine does not modify dorsal horn touch-evoked allodynia in the mononeuropathic rat: a Fos study. Pain 2001;92:389-398.

12. Hao S, Takahata O, Mamiya K, Iwasaki H. Sevoflurane suppresses noxious stimulus-evoked expression of Fos-like immunoreactivity in the rat spinal cord via activation of endogenous opioid systems. Life Sci 2002;71:571-580.

13. Yaksh TL. Behavioral and autonomic correlates of the tactile evoked allodynia produced by spinal glycine inhibition: effects of modulatory receptor systems and excitatory amino acid antagonists. Pain 1989;37:111-123.

14. Sorkin LS, Puig S, Jones DL. Spinal bicuculline produces hypersensitivity of dorsal horn neurons: effects of excitatory amino acid antagonists. Pain 1998;77:181-190. 
15. Hao JX, Xu XJ, Wiesenfeld-Hallin Z. Intrathecal gammaaminobutyric acidB (GABAB) receptor antagonist CGP 35348 induces hypersensitivity to mechanical stimuli in the rat. Neurosci Lett 1994;182:299-302.

16. Smith GD, Harrison SM, Birch PJ, et al. Increased sensitivity to the antinociceptive activity of $(+/-)$-baclofen in an animal model of chronic neuropathic, but not chronic inflammatory hyperalgesia. Neuropharmacology 1994;33:1103-1108.

17. Hwang JH, Yaksh TL. The effect of spinal GABA receptor agonists on tactile allodynia in a surgically-induced neuropathic pain model in the rat. Pain 1997;70:15-22.

18. Malan TP, Mata HP, Porreca F. Spinal GABA(A) and GABA(B) receptor pharmacology in a rat model of neuropathic pain. Anesthesiology 2002;96:1161-1167.

19. Ibuki T, Hama AT, Wang XT, et al. Loss of GABAimmunoreactivity in the spinal dorsal horn of rats with peripheral nerve injury and promotion of recovery by adrenal medullary grafts. Neuroscience 1997;76:845-858.

20. Glorioso JC, Fink DJ. Herpes vector-mediated gene transfer in treatment of diseases of the nervous system. Annu Rev Microbiol 2004;58:253-271.

\section{An LRRK2 Mutation as a Cause for the Parkinsonism in the Original PARK8 Family}

Manabu Funayama, PhD, ${ }^{1}$ Kazuko Hasegawa, MD, PhD, ${ }^{2}$ Etsuro Ohta, MMSci, ${ }^{1}$ Noriko Kawashima, MD, ${ }^{3}$ Masaru Komiyama, BS, ${ }^{1}$ Hisayuki Kowa, MD, PhD, ${ }^{4}$ Shoji Tsuji, MD, PhD, ${ }^{5}$ and Fumiya Obata, $\mathrm{PhD}^{1}$

\begin{abstract}
We detected a missense mutation in the kinase domain of the $L R R K 2$ gene in members with autosomal dominant Parkinson's disease of the Japanese family (the Sagamihara family) who served as the basis for the original defining of the PARK8 Parkinson's disease locus. The results of the Sagamihara family, in combination with the unique pathological features characterized by pure nigral degeneration without Lewy bodies, provided us with valuable information for elucidating the protein structure-pathogenesis relationship for the gene product of LRRK2. We did not detect this mutation or other known mutations of the LRRK2 gene in Japanese patients with sporadic Parkinson's disease.
\end{abstract}

Ann Neurol 2005;57:918-921

Parkinson's disease (PD) [MIM 168600] is one of the most common neurological disorders and is characterized by rigidity, bradykinesia, tremor, and postural instability. Although PD is a sporadic disease in most cases, various hereditary forms presenting clinical phenotypes similar to those of sporadic PD have been recognized. Molecular genetic studies on these familial forms of parkinsonism have substantially advanced our understanding of the molecular pathogenesis of this disease. ${ }^{1-6}$

In a previous study, we identified a new locus for PD, PARK8 (12p11.2-q13.1), by genome-wide linkage

From the ${ }^{1}$ Division of Clinical Immunology, Kitasato University Graduate School of Medical Sciences; ${ }^{2}$ Department of Neurology, National Hospital Organization, Sagamihara National Hospital, Sagamihara; ${ }^{3}$ Kawashima Neurological Clinic, Fujisawa; ${ }^{4}$ Kitasato University, Sagamihara; and ${ }^{5}$ Department of Neurology, Tokyo University, Tokyo, Japan.

Received Dec 27, 2004, and in revised form Mar 11, 2005. Accepted for publication Mar 11, 2005.

Current address for Dr Funayama: Research Institute for Diseases of Old Ages, School of Medicine, Juntendo University, Tokyo, Japan.

Published online May 4, 2005 in Wiley InterScience (www.interscience.wiley.com). DOI: 10.1002/ana.20484

Address correspondence to Dr Obata, Department of Immunology, Kitasato University School of Allied Health Sciences, 1-15-1 Kitasato, Sagamihara, Kanagawa 228-8555, Japan.

E-mail: obata@ahs.kitasato-u.ac.jp 